M. Cherif Bassiouni (ed.)

\title{
The Islamic Criminal Justice System
}

Oceana Publications Inc. London, Rome, New York, $1982 \$ 30,-$

Das Buch dokumentiert die "1. Internationale Konferenz über den Schutz der Menschenrechte im Islamischen Strafrechtssystem", die im Mai 1979 in Syracus abgehalten wurde; es enthält neben acht Beiträgen, die aus den Konferenzvorträgen ausgewählt wurden, auch drei selbständige Aufsätze und im Anhang einen zusammenfassenden Bericht über die Konferenz sowie die Schlußresolution der Konferenz über die grundlegenden Rechte des Beschuldigten im Strafverfahren.

Nach Angaben des Verlages ist das vorliegende Buch die erste umfassende Darstellung des Islamischen Strafrechtssystems in englischer Sprache. Das wäre an sich schon sehr verdienstvoll, es kommt aber noch ein Charakteristikum hinzu, das diesem Buch einen besonderen Reiz verleiht und es zu einer geradezu faszinierenden Lektüre werden läßt: Alle Autoren, die sämtlich islamische Rechtsgelehrte sind, begnügen sich nicht damit, das islamische Strafrechtssystem darzustellen, sondern sie alle bemühen sich - mehr oder weniger intensiv - zu beweisen, daß das islamische Strafrechtssystem ohne weiteres mit dem modernen Menschenrechtssystem in Einklang stehe. Auch wenn das islamische Rechtssystem selbst überzeitlich und unverändert gültig sei, so sei seine Anwendung offen für Weiterentwicklung, und damit könne es auch heutigen Anforderungen genügen (vgl. Bassiouni, Introduction, S. XIV). Doch ist diese Sicht meines Erachtens zu optimistisch. Zwar gibt es weitreichende Parallelen zwischen dem islamischen Rechtssystem und dem Menschenrechtssystem hinsichtlich der Stellung des Beschuldigten im Strafverfahren, wie auch die Autoren zu unterstreichen nicht müde werden, aber dabei wird der grundsätzliche Unterschied im Verständnis der Menschenrechte vernachlässigt: Nach islamischem Verständnis können Menschenrechte nur so weit anerkannt werden, wie sie dem Islam nicht widersprechen. Daraus ergeben sich aber durchaus Schwierigkeiten, denn nicht alle Regelungen der Scharia entsprechen dem herrschenden Menschenrechtsverständnis. An solchen Punkten sind dann auch die Harmonisierungsversuche der Autoren mitunter nur bedingt nachvollziehbar. So mag das Argument, die in der Scharia festgelegte unterschiedliche Rechtsstellung der Muslime gegenüber Nicht-Muslimen in bestimmten Bereichen sei lediglich in administrativer und politischer Hinsicht verschieden, einen Muslim überzeugen, beim nicht muslimischen Leser löst diese Argumentation Bedenken aus. Bei der Lektüre des Buches begegnen dem Leser fortwährend solche "Bruchstellen", deren "Bewältigung" durch die Autoren allein aus islamischer Sicht zu befriedigen vermag. Ein weiteres eklatantes Beispiel für dieses Auseinanderklaffen westlicher und islamischer Menschenrechtsvorstellungen bildet die Religionsfreiheit; sie gehört zwar zu den grundlegenden Dingen, die der Islam allen Menschen garantiert (AlSalem, S. 56), aber sie umfaßt nach islamischer Auffassung (für Muslime) nicht das Recht zum Wechsel des religiösen Bekenntnisses, denn das gilt als Apostasie (Ridda), auf die die Todesstrafe steht.

Aus allen Beiträgen geht aber hervor, daß es auch weitaus restriktivere Ansichten unter 
islamischen Rechtsgelehrten gibt. Man kann sogar im Vergleich der Beiträge Unterschiede hinsichtlich der Bewertung einzelner Probleme feststellen. Grundsätzlich aber liegen alle Autoren auf einer Linie, die ich als "moderne Orthodoxie" bezeichnen möchte - "modern", weil sie sich sehr nachhaltig bemühen, das islamische Recht, das ja unter ganz anderen sozio-kulturellen Bedingungen entstand, heutigen Erfordernissen anzupassen, "orthodox", weil sie den unmittelbaren Geltungsanspruch der Scharia nicht in Frage stellen, sie also insbesondere nicht auf die ihr innewohnende Ethik reduzieren wollen. Auch die juristische Argumentation ist sehr islamisch: Als Belege werden zahlreiche Koranverse angeführt, die oftmals gar nicht einschlägig erscheinen, aber in der Rechtstradition des Islam den Wert von Argumenten haben; ferner zitieren viele Autoren ausführlich die Äußerungen berühmter Rechtsgelehrter, die oftmals in der Frühzeit des Islam gelebt haben. Das ist anfangs für die Leser etwas gewöhnungsbedürftig, gibt aber zugleich sehr aufschlußreiche Einblicke in das islamische Rechtsdenken und die islamrechtliche Argumentationstechnik.

Insgesamt hält das Buch weit mehr als es verspricht: es bietet nicht nur eine Einführung in das Islamische Strafrecht, sondern gewährt auch Einblicke in die Methodenlehre des Islamischen Rechts, und darüber hinaus ist es eine ausgezeichnete, authentische (!) Informationsquelle über das Menschenrechtsverständnis im Islam.

Ulrich Deffaa

\section{Ernst E. Hirsch}

\section{Rezeption als sozialer Prozeß. Erläutert am Beispiel der Türkei}

Schriftenreihe zur Rechtssoziologie und Rechtstatsachenforschung, hrsgg. von Ernst E. Hirsch und Manfred Rehbinder, Band 50; Duncker \& Humblot, Berlin 1981, 139 S., DM 58,--

In dem vorliegenden Band gibt Hirsch Einblick in den Rezeptionsprozeß des schweizerischen Zivilgesetzbuches (ZGB) und Obligationenrechts (OR) sowie die Kodifikation des Handelsgesetzbuches (HGB) von 1926 und 1956 in der Türkei, der seinen Anfang im Zerfall des Osmanischen Reiches mit Ende des Ersten Weltkrieges und dem Friedensvertrag von Lausanne vom 24. 7. 1923 nahm, in dem sich die Türkei zur Reorganisation ihres bis dahin islamisch bestimmten Rechts- und Gerichtswesens verpflichtete.

Wie der Titel verdeutlicht, versteht Hirsch Rezeption nicht als einen "einmaligen Akt der Gesetzgebung (Pritsch) (S. 11), sondern als einen Prozeß exogenen "sozialen Wandels" (S. 12), in dem sich die "Akkulturation" fremden Rechts im übernehmenden Lande vollzieht. Mit der juristischen Inkraftsetzung rezipierten Rechts ist danach noch keine Rechtsordnung gebildet, sondern "lediglich der Plan zu einer solchen" (S. 13), der zu seiner Verwirklichung der Akzeptanz in allen gesellschaftlichen Gruppen und Schichten bedarf, begründet. Daß es dabei nicht nur in kulturell so unterschiedlichen Systemen wie der Türkei und der Schweiz, sondern vor allem beim Hinzutreten ganz unterschiedlicher 